Manuel Correia M.

Profesor de la Facultad de Filosofía

Pontificia Universidad Católica de Chile

\title{
Libertad humana y presciencia divina en Boecio
}

En su Libro V de la Consolación de la Filosofía, Boecio examina uno de los problemas más complejos que la filosofía postaristotélica encuentra: el de la compatibilidad de la libertad humana y la presciencia divina (divina praescientia). ¿Es el hombre libre? Boecio hace de este problema un tratamiento de gran valor teórico para la filosofía posterior, especialmente para la filosofía cristiana, encontrándose, de diverso modo, vestigios de su discusión y solución, en pensadores posteriores tan distintos como Sto. Tomás de Aquino, Lorenzo Valla y Leibniz. Así, Boecio junto con San Agustín (de quien no parece depender en su solución y discusión del problema) se convierten en los filósofos fundamentales de la metafísica cristiana de la libertad humana por sus contribuciones a este problema inmenso. En este artículo voy a intentar mostrar que la solución de Boecio es una elaborada pieza de doctrina neoplatónica, originalmente premunida de instrumental lógico tomado desde Aristóteles, que se inscribe en la línea de dificultades y discusión desarrollada por los estoicos, y que logra contribuir efectivamente en la clarificación del problema al poner la noción de razón (ratio) como condición esencial para la comprensión de la naturaleza de la libertad humana (y de toda creatura racional). Voy a indicar de tiempo en tiempo cómo esta solución dirige definitivamente la discusión metafísica medieval y moderna, al menos hasta Leibniz, sugiriendo así (sin pretender una historia completa de esta discusión) que la solución de Boecio está en la base de toda otra solución que presuponga una noción de razón. Para apreciar más su aporte, comenzaré haciendo una breve historia del problema, desde su origen en la filosofía griega.

\section{ORIGEN DEL PROBLEMA}

Una de las evidencias textuales más sorprendentes que nos entrega la historia de la filosofía occidental, es el hecho, cada vez más admitido, de que el problema de la libertad humana es postaristotélico (1). Esto significa desde luego que ninguno de

(1) "The problem of determinism and responsibility was not realized, in the form in which it was eventually passed on to post-classical thinkers, until relatively late in the history of Greek thought". Cf. R.W. Sharples, Alexander of Aphrodisias on Fate, p. 3, London 1983: Duckworth. 
los presocráticos, ni Platón ni Aristóteles, advirtieron en sus escritos que la libertad humana era un problema filosófico primero y primordial, tal como la filosofía medieval y moderna posteriormente evidenciarían (2). Las evidencias textuales nos muestran que Epicuro (341-270 a. C.) es el primero en poner en cuestión el hecho de si el hombre es o no libre, y el primero en poner la dilucidación de esta cuestión como fundamental para el éxito de toda filosofía del hombre y de la naturaleza (3). En efecto, Epicuro se opone a las concepciones atomistas de Demócrito y Leucipo, precisamente porque en este sistema atomista riguroso la libertad humana y el azar no tienen lugar, sino que todo se explica por determinación de posición y movimiento de los átomos. La parénclisis o clinamen, como un movimiento azaroso de los átomos, se convierte, así, en lo distintivo de la física de Epicuro, y en la posibilidad de recuperar la libertad humana y presentar un sistema atomista más acorde con la intuición, que muchas veces nos presenta a los hombres como actuando por causas propias o simplemente sin causa, sujetos al más puro de los azares.

Después de Epicuro la cuestión de la libertad humana se convierte en un tema obligado de la filosofía occidental. Así los estoicos van a tomar nuevamente el problema y a ofrecer distintas soluciones. La motivación de los estoicos era similar a la de Epicuro, aunque no igual, ya que si para Epicuro era un problema la hipótesis de un sistema atomista determinístico, para los estoicos el problema era la compatibilización del lógos cósmico, que era naturaleza y sabiduría, y al cual nada se le sustraía, con los movimientos contingentes y libres del hombre. Y esto es así, porque para los estoicos crece la evidencia de que la naturaleza es un sistema de causas y efectos que, como una ley, se ejerce sobre cada una de las cosas. Así, todas y cada una de las cosas están gobernadas por el destino o heimarméne (la ley de causas y efectos), y no habiendo más ley que esta, el azar, la casualidad y la suerte no existen (4). La solución de la primera generación de estoicos fue la de que somos libres si podemos vivir acordes con la razón cósmica, pues adonde fuera ella, allí el hombre

(2) Sin embargo, el que Aristóteles en De Int. 9 quiera objetar una posición determinista es, por supuesto, una excepción atendible, especialmente por el uso de este texto en posteriores discusiones teológicas asociadas al problema de la libertad y la presciencia divina. También merece atención su discusión sobre el azar (to automáton) y la suerte (tyché) en Física II, 4-6, y sus alusiones al hombre libre (ánthropos eleútheros) de Metafísica I, 2, 982b25. Pero ninguno de estos textos revela que, en su opinión, la solución de este problema debe ser anterior a toda otra preocupación filosófica. Por ello, parece más razonable la tesis de que Aristóteles, en general, no hace de la cuestión de si el hombre es libre o no una cuestión central de su filosofía.

(3) En un fragmento conservado por Diógenes de Enoanda (32, Chilton), se encuentra lo siguiente: "Si alguno utiliza la argumentación de Demócrito, afirmando que no hay ningún movimiento libre en los átomos, a causa de su choque mutuo, de donde se deduce que todo se mueve forzosamente, le replicaremos: ¿no sabes tú, quienquiera que seas, que hay también en los átomos un movimiento libre que Demócrito no ha descubierto, pero que Epicuro ha traído a la luz: la existencia de la declinación (parénklisis), como lo muestra a partir de los fenómenos". Diogenes Oenoandensis fragmenta, ed. C.W. Chilton (Leipzig, 1967: Teubner eds.) Cf. también Cicerón de Fato 22, 23: "Epicuro introdujo dicho concepto temiendo que si el átomo se movía siempre por efecto de una gravedad natural y necesaria, no quedaría en nosotros libertad alguna, por cuanto el espíritu se movería entonces según el movimiento obligatorio de los átomos. Demócrito, el inventor de los átomos, prefirió admitir que todo sucede necesariamente, antes de despojar a los corpúsculos indivisos de su movimiento natural". (H. Rackham, 1942, Loeb Classical Library).

(4) Es importante notar cómo esta tesis contrasta con la tesis de Aristóteles en Física II, 4-6. 
sería más feliz y libre, mientras que infeliz y esclavo si se oponía y resistía a la heimarméne (5):

"Ellos probaron que todo proviene de la heimarméne, valiéndose de la siguiente comparación: si un perro, estando atado a un carro, quiere seguir al carro, avanza con él y es llevado, y de este modo hace lo que quiere, y al mismo tiempo ejecuta aquello a que está forzado; pero si no quiere obedecer, se verá forzado a hacer cuanto los hados han dispuesto".

Crisipo (ca. 280-207 a. C.), posteriormente, va a introducir una importante diferencia al distinguir la necesidad de la naturaleza y el carácter del hombre con el fin de encontrar un lugar a la responsabilidad de nuestras acciones. En su conocido símil del cilindro, nos pone en esta distinción: si un cono y un cilindro son echados a rodar en una pendiente, ambos rodarán por necesidad -pues así opera la naturaleza en los hombres- pero cada cuerpo, el cilindro y el cono, rodará de distinto modo: tal es así porque cada hombre, como cada cuerpo aquí, tiene un carácter propio, el cual se debe no a la sola naturaleza, sino a sí mismo. Con este argumento Crisipo reconoce que hay un ámbito de cuestiones que dependen de nuestro ser más que de la naturaleza, y que, por tanto, la naturaleza no ejerce una determinación directa sobre cada una de las cuestiones que nos atañen como humanos, sino que indirecta, tal como el cono y el cilindro conservan cierta independencia frente a la necesidad de rodar por la pendiente. Probablemente entonces la lección que sacaba Crisipo era que no se debía desatender nuestros asuntos propios, ya que no era lo mismo dejar que las cosas sucedieran que hacer algo por cambiarlas. Esto es precisamente lo que sostiene en contra de otro argumento que nos ha sido conservado: el así llamado 'argumento perezoso' (árgos lógos) (6).

"Si está destinado que tú te vayas a sanar, te sanarás ya sea que visites al doctor o no lo visites; si está destinado que tú no te sanarás, no te sanarás ya sea que llames al doctor o no lo llames; de modo que una y la otra cosa está destinada; así, en cualquier caso, no tiene sentido que llames al doctor".

El estoicismo romano posteriormente adoptará esta distinción de Crisipo. Es así como vemos en Epicteto, Séneca y Marco Aurelio una clara noción de la antinomia entre libertad y determinismo, pero al mismo tiempo una clara defensa de la existencia de la libertad al interior del hombre como un espacio que no puede ser coaccionado desde el exterior. La libertad se da en lo que depende de nosotros (to eph' hemin). Así es patente en el estoicismo romano una marcada división entre dos ámbitos de la vida humana, el interior donde radica la libertad y el exterior que está fuera de la tutela del hombre. Al mismo tiempo y en acuerdo con esto se aprecia

(5) Hipólito Romano, Refutatio omnium haeresium 21, A, II, 975; GCS XXVI, P. Wendland (ed.), Leipzig 1916. Cf. también E. Elorduy, El Estocismo, vol. i, p. 141, Madrid 1972: Gredos.

(6) Stoicorum Veterum Fragmenta (=SVF) 2.956 (=Cicerón De fato 30), H. Von Arnim (ed.), Stuttgart 1968: Teubner. 
también una tensión entre estos ámbitos: la de conservar el ámbito interior y despreciar lo de lo exterior. Por ejemplo, Epicteto dice así (7):

"De las cosas, algunas están en nuestro poder, y otras no. En nuestro poder están la opinión, la tendencia hacia algo, el deseo, la aversión y, en una palabra, cualquier cosa que dependa de nuestros actos propios: pero no están en nuestro poder el cuerpo, la propiedad, la reputación, el poder y, en una palabra, cualquier cosa que no dependa de nuestros propios actos. Y las cosas que están en nuestro poder son por naturaleza libres, no sujetas a impedimentos o trabas. Pero las cosas que no están en nuestro poder son débiles, esclavas y sujetas al poder de otros".

Hay que notar que para estos estoicos lo exterior al hombre no es solo aquello que ocurre en la naturaleza, los eventos físicos que existen y se relacionan sin depender de nuestra voluntad, sino también las cuestiones que siendo humanas se originan en las pasiones, especialmente el deseo y el temor. Estas pasiones son el principal impedimento de la libertad humana interior. Con los estoicos romanos, pues, surge aquel elemento literario de la esclavitud de las pasiones sobre la razón, un tema que más tarde va a recuperar Spinoza en su discusión sobre la naturaleza humana y la libertad. Y que iba a ser examinado más finamente por Hobbes, Locke y Hume posteriormente. Se es libre si se disuelven las pasiones que nos avasallan y se conserva ese 'sí mismo' de que habla Marco Aurelio (8). Al refrenar su imperio accedemos a la verdad y al bien, a la realidad y a la norma moral que está inscrita en la naturaleza como todos los estoicos creyeron. La virtud pues consiste en llegar a ser libres terminando con el dominio de las pasiones. Así, tal como en la vieja Stoa, la libertad llega a ser algo que solo el sabio posee y es opuesta normalmente a la esclavitud (e.g. diss II, 2.13) y es atribuida más que a las acciones, al estado de ánimo. Ello es lo propio del sabio, quien vive de acuerdo con un estado de ánimo donde reina la tranquilidad de mente, la ataraxia, y la apatheia.

Según lo que me interesa destacar en este trabajo, tanto la conciencia de la dificultad del problema de la libertad humana como la profundidad de los intentos de solución comienzan un desarrollo en la filosofía postaristotélica, especialmente cristiana, en que paulatinamente se va delimitando y conociendo mejor la naturaleza de la razón humana. En palabras simples puede decirse que mientras más profunda y convincente es la solución al problema de la libertad humana tanto más profunda y convincente es su noción de razón. Así se ve cómo para los estoicos, mucho más que para Epicuro, la posibilidad de la libertad se ve en relación a la posibilidad de que exista algo que pueda ser llamado to eph' hemin, 'lo que depende de nosotros', como algo no solo que está en nuestro poder hacerlo, como parece haber sido mantenido por la primera Stoa, sino también como algo diferente de las pasiones que sojuzgan nuestra verdadera razón libre, lo que parece haber sido la contribución de los estoicos romanos.

(7) Epictetus, Enchiridion, tr. G. Long, New York 1955: Prometheus Books

(8) Cf. Marco Aurelio, Meditaciones, VI, 8; VII, 16, 28; VIII, 2, 48; IX, 4; XI, 1, 12, et passim (Madrid 1990: Gredos). 
Es en esta línea que la discusión del problema por Boecio en el libro $\mathrm{V}$ de su Consolatio Philosophiae hace una contribución muy importante, lo cual se aprecia más, creo, por la influencia que su examen ejerció no solo durante la Edad Media, sino también durante la Edad Moderna.

Antes de pasar a ver cómo esto se perfila aún más en Boecio, debo decir una palabra sobre San Agustín. En De libero arbitrio y en algunos pasajes de otras obras San Agustín se encarga del problema de si o no la libertad humana se opone a la presciencia y providencia divinas, y su examen es igualmente muy influyente en la metafísica cristiana medieval y moderna. En san Anselmo hay vestigios marcados de su presencia, y en Santo Tomás de Aquino y Leibniz se aprecia claramente el eco de las argumentaciones que Agustín hace a favor de la libertad apelando a la gloria de Dios: si no fuéramos libres la gloria de Dios no sería esa superlativa que le atribuimos. Dios en efecto prefiere a un hombre que peca libremente a uno que no peca por necesidad y determinación impuesta, del mismo modo que para él es más valioso una criatura libre que puede pecar que una que es buena porque no puede pecar (9). Sin embargo, las evidencias para creer que la discusión de Agustín sobre la libertad humana haya sido adoptada por Boecio son escasas y débiles, y ello es lo que ha levantado la polémica de Courcelle de que Boecio ni siquiera leyó los primeros Diálogos de Agustín (10). Si San Agustín es una fuente de la especulación metafísica que Boecio hace en el libro V de la Consolatio, es una cuestión polémica, y de este modo no parece conveniente que lo incluyamos en esta historia del problema con que he introducido la discusión en Boecio, aunque reconozcamos desde un principio la importancia de su especulación en este tema (11).

\section{LAS FUENTES DE LA DISCUSIÓN DE LA LIBERTAD HUMANA EN LA CONSOLATIO V DE BOECIO}

En la Consolatio Boecio muestra enteramente sus opiniones en filosofía. Es un neoplatónico con marcada influencia de Porfirio, Yámblico y Proclo. Y ello quiere decir que es un neoplatónico que adopta todo tipo de instrumentos lógicos de tipo aristotélico. Si la lectura de su comentario sobre De Interpretatione 9 (12), de

(9) De Libero arbitrio, III, 56, p. 359 (=CSEL 74, 103), en Obras de San Agustín, BAC, Madrid 1982: La Editorial Católica. "[...] así es más excelente la criatura que peca por su propia y libre voluntad que aquella otra que es incapaz de pecar por carecer de voluntad libre".

(10) Cf. Courcelle, P., "Le Personage de Philosophie dans la literature latine", Journal des Savants (1970), 209-52.

(11) Sobre una posible influencia de San Agustín en Boecio, ver: E.T. Silk, "Boethius's Consolatio Philosophiae as a sequel to Augustine's Dialogues and Soliloquia", en Harvard Theological Review xxxii (1939), pp. 19-30. También A. Crabe, "Literary Design in the De Consolatione Philosophiae", en Boethius. His Life, Thought and Influence, M. Gibson (ed.), Oxford 1981, pp. 237-277: Blackwell. Una opinion contraria tiene, sin embargo, H. Chadwick: "It is possible to draw up a considerable list of anticipations in Augustine's writings, though none where one can establish a verbal echo or the probability of a literary dependence. Perhaps the closest analogies occur in Augustine's Soliloquies which, like Boethius, speak of the wings of the soul (Sol. I, 14, 24; II. I; 20, 34 f.). Cf. Chadwick, p. 11, en Gibson (1981).

(12) MEISER (1877-1880): Anicii Manlii Severini Boetii Commentarii in Librum Aristotelis PERI ERMHNEIAS. Prima et secunda editio. C. Meiser (Ed.), Teubner Eds., Leipzig 1877-1880. Para 
Aristóteles, dejaba la impresión de que Boecio se identificaba con la enseñanza de los peripatéticos en contra del determinismo estoico, y si a lo largo de sus comentarios las interpretaciones platonizantes de la lógica de Aristóteles son inexistentes, en la Consolatio no deja ninguna duda de su compromiso metafísico con el neoplatonismo, y ello permite entonces entender que en sus comentarios sobre la lógica de Aristóteles se aleja de las interpretaciones estoicas y neoplatonizantes de la tradición comentarística, para rescatarla en su pureza genuina, de modo que sirvan efectivamente para usos metafísicos y teológicos propios de su neoplatonismo y cristianismo (tal como se ve esto último en sus Tratados Teológicos). De hecho, de estos tres maestros neoplatónicos mencionados, Boecio es el más encandilado por la utilidad de la lógica de Aristóteles, pero ello es así, como la Consolatio muestra, para asimilar el Aristóteles lógico completamente en la teología platónica (13).

En efecto, el De Interpretatione 9 de Aristóteles, traducido y comentado por Boecio antes de su Consolatio, discute si las proposiciones singulares con verbo en futuro pueden determinarse como verdaderas o falsas, tal como todo el resto de especies de proposición allí distinguidas. Aristóteles allí objeta a los que afirman que esta determinación es posible, y con ello una suerte de determinismo lógico sobre los hechos del mundo. Pero si la refutación del determinismo era la tarea del comentario de Boecio, ahora en la Consolatio, yendo más allá de Aristóteles, tiene que salvar la realidad de la libertad humana y de la contingencia en la perspectiva de una presciencia divina que conoce todo infaliblemente. Y ello no lo puede hacer con Aristóteles, quien no se planteaba esta suerte de problemas.

Por tanto, la Consolatio V es un ejemplo de incorporación de la lógica de Aristóteles en la teología neoplatónica de la libertad y sus problemas. Un hito ejemplar de la forma de pensamiento neoplatónico del siglo VI d. C., que se puede ver también en Amonio Hermeias cuyo trabajo sobre De Int. 9 es totalmente independiente de los de Boecio (14). Y en este sentido, creo, debe interpretarse ese ideal incumplido de traducir al latín todos los textos de Aristóteles y Platón que llegaren a sus manos (in Int. 2, 79-80) y el deseo también incumplido de mostrar que Platón y Aristóteles no estaban en contradicción, sino en armonía (in Int 2, 79, 16 ss.), el cual había sido ya anunciado por Porfirio, pero que Boecio reivindica como propio en su comentario al De Int. de Aristóteles.

efectos de abreviación de esta obra utilizamos 'in Int' para el primer comentario, e 'in Int. 2' para el segundo.

(13) Conviene recordar aquí lo que el discípulo de W. Jaeger, F. Solmsen, ha dicho respecto de este tiempo y la asimilación de la lógica aristotélica por los neoplatónicos: "This is the time when the Neoplatonists became the guardians not only of pagan intellectual civilization in general but also of the Aristotelian legacy in particular". Solmsen, F., "Boethius and the History of the Organon", en American Journal of Philology, (1944), vol. 65, p. 72.

(14) La cuestión de las fuentes de los comentarios lógicos de Boecio ha sido muy discutida durante la segunda mitad del siglo XX y aún hasta hoy carece de una respuesta definitiva. Para un recuento de esta discusión, ver M.A. Correia "Boecio y Amonio sobre Peri Hermeneias 16b14-15: el verbo indefinido en Aristóteles”, pp. 120-121, n.25, en Diadokhé, vol. 1, N¹-2, 1998. Y ver también el reciente juicio de R. Sorabji en "Boethius, Ammonius and their different Greek backgrounds", en Ammonius On Aristotle On Interpretation 9 with Boethius On Aristotle On Interpretation 9, pp. 16-17; Tr. D. Blank \& N. Kretzmann, en Ancient Commentators of Aristotle, R. Sorabji (Ed.), London 1998: Duckworth 


\section{LA DOCTRINA DE LA LIBERTAD DE BOECIO}

El problema de si puede existir una verdadera libertad humana en el hombre cuando se acepta la existencia de una mente divina que es omnisciente y providente es solucionado por Boecio afirmando que la libertad existe de hecho porque el hombre es racional, aunque ello no garantiza que vivamos en libertad. La tesis recuerda esa de Rousseau en el capítulo primero del Contrato Social cuando dice "El hombre ha nacido libre, pero vive en todas partes encadenado". La fundamentación de esta tesis hecha por Boecio en la Consolatio V es una contribución a la historia de la discusión empezada por Epicuro que es calificada por Boecio mismo como novedosa y más acabada que la de ningún otro autor en el pasado. (V, 4p, 1-9). La dama Filosofía, que aparece en su celda de encierro y muerte para reeducarlo en la verdadera doctrina, alude implícitamente a Cicerón y su "solución" de quitarle presciencia a Dios para asegurar la libertad humana, como una solución insuficiente. La doctrina verdadera es expuesta por Boecio en dos partes, en la primera se expresa dogmáticamente acerca de qué es libertad de arbitrio y cómo es posible en el hombre. En la segunda parte que va hasta el final del libro se examinan las presuntas dificultades que tiene una tesis así.

$\mathrm{Su}$ aspecto positivo reluce en el comienzo del libro V (I, 5-29) cuando Boecio pone la razón como causa y condición de la libertad. Ello no solo significa que no hay ninguna creatura que sea racional y que esté privada de libertad, sino también que ella es más libre cuanto más racional pueda ser (15):

"Sí -me contestó-. Existe el libre arbitrio (libertas arbitrii). No hay un ser dotado de razón falto de libertad. Todo aquel que por su misma naturaleza puede servirse de la razón tiene la facultad de poder juzgar y discernirlo todo. Por sí mismo distingue lo que ha de rechazar y lo que ha de elegir. Cada uno busca lo que estima apetecible y huye de lo que considera rechazable. Así, quien está dotado de razón tendrá también la libertad de querer o no querer, si bien advierto que no todos los seres tienen el mismo grado de libertad".

Conforme a la doctrina que Boecio enseña, hay libertad y también grados de libertad, ya que no todos los seres racionales tienen el mismo grado de razón. Los seres celestiales que gozan de una penetración mental (perspicax iudicium) mayor, y una voluntad incorrupta, junto con la habilidad de lograr lo que quieren, son máximamente libres, y lo son así porque su razón los lleva a la contemplación de la mente divina, quien, como se colige desde el texto, es eternamente libre, inteligente y buena.

Es así que la contemplación de esta mente divina es lo que hace plenamente libre al hombre, tanto cuanto su razón se lo permita (V, II, 14-27):

"Los seres celestiales y divinos poseen un juicio clarísimo, una voluntad inquebrantable y un poder eficacísimo para llevar a cabo sus deseos. Los seres humanos, en cambio, son necesariamente tanto más libres cuanto más se apli-

(15) Para esta y las siguientes traducciones de la Consolatio de Boecio utilizo, con pequeñas modificaciones, la traducción castellana de Pedro Rodríguez Santidrián, en Boecio La Consolación de la Filosofía, Madrid 1999: Alianza Editorial. 
can a la contemplación de la mente divina, y tanto menos libres cuanto más descienden a los seres materiales. Y todavía menos cuando quedan atrapados en las redes de la tierra. Alcanzan, por último, la máxima esclavitud cuando se entregan al vicio y pierden la posesión de su propia razón. Sucede que cuando han apartado sus ojos de la luz de la verdad superior para fijarlos en el mundo inferior y tenebroso, se ven enseguida turbados por pasiones funestas $\mathrm{y}$, al ceder a ellas y consentirlas, no hacen más que fomentar la esclavitud contraída, haciéndose, por decirlo así, prisioneros de su libertad".

La doctrina positiva o dogmática mencionada de entrada por Boecio asegura que el hombre es libre por ser racional, si bien ello no garantiza que viva libremente, tal como el hecho de poseer razón no nos garantiza que actuemos racionalmente siempre y en todo caso. La influencia de esta doctrina puede verse de distinto modo en autores medievales como Santo Tomás de Aquino, cuando afirma que Dios es el ser más libre de todos los que existen (16), en el renacentista Lorenzo Valla, quien narra el mito de Sexto Tarquinio (17), y en algunos modernos como Spinoza y Leibniz, cuando el primero hace de Dios causa sui y pone esta condición la única para ser libre (18), y el segundo cuando afirma que Dios para crear este mundo no sigue más ley que la de lo mejor posible, la cual consta en su misma naturaleza simple (19). Por lo demás, dicho en general, la doctrina aquí expresada por Boecio es la de todos aquellos que ponen a la verdad por sobre la libertad, en acuerdo con la enseñanza cristiana de "Conoceréis la verdad y la verdad os hará libres" (Jn 8, 32) (20).

\section{LAS DIFICULTADES}

Esta doctrina, sin embargo, no hace evidente cómo esta libertad humana puede convivir y ser compatible con una mente divina que conoce todas las cosas y puede prever qué es lo que ocurrirá y no ocurrirá. Y este, como ya había sido en el pasado, es el problema que toda doctrina de la libertad tiene que resolver.

Así, Boecio enfrenta un problema que ya ha hecho cierta tradición, pero ya no es el Lógos en la Stoa el que entra en conflicto, sino el dios bonus rector del neoplatonismo. Este dios del platonismo tiene también sus dificultades. Aristóteles aquí parece irreconciliable con el neoplatonismo, no solo porque el dios de Aristóteles no parece conocer los singulares, sino también por su noción de azar. La discusión de Boecio parte desde aquí, por redefinir o interpretar la noción de azar en Aristóteles con una

(16) Santo Tomás de Aquino, Summa Contra Gentiles, I, LXXXVIII (Leoninus), Roma 1961: Marietti.

(17) Cf. L. Valla, Dialogue sur le libre arbitre, tr. e introd. por Jacques Chomarat, Paris 1983: J. Vrin. El mito de Sexto Tarquinio tuvo una notable repercusión en autores modernos como Leibniz, quien lo cita en su Teodicea, $\$ 405$ y ss.

(18) "God alone is a free cause; for God alone exists and acts from the necessity alone of his own nature" (Ethica I, Prop. XVII, Cor. II). Ver también Prop. XVII, Dem. y Cor. I. (Spinoza. Ethics, tr. G.H.R. Parkinson, London 1989: Everyman's Library). En este respecto, cf. el interesante comentario de H.F. Hallett (Benedict de Spinoza. The Elements of his Philosophy, p. 28; Bristol 1957/1990: Thoemmes).

(19) GW Leibniz, Discourse de Métaphysique, 2, 3; C.I. Gerhardt (ed.) Die Philosophischen Schriften von Leibniz, vol. IV Berlin: Weidmann, 1875-90; reprinted Hildesheim: Olms, 1965

(20) Por ejemplo, Juan Pablo II, Veritatis Splendor, cap. II, nn. 31-53. Santiago de Chile 1993: San Pablo. 
reflexión en el marco del dios providente, infalible. Para Aristóteles el azar, la casualidad y la suerte no son causas de la physis, pero existen (21). A diferencia de los estoicos, para quienes un mero accidente llenaba el todo de variaciones, de tal modo que el mundo es un sistema donde el azar, la casualidad y la suerte no tienen lugar, para Aristóteles el movimiento accidental, libre, casual, y a veces azaroso de los cuerpos, era un hecho que convivía con la causalidad de otros fenómenos. (Así parece que esta visión de mundo heredada pudo evitar que Aristóteles pensara el problema de la libertad como primordial). Boecio como neoplatónico tiene que transformar esta visión del azar aristotélico (22), pero allí mismo brota el problema: si existe un Dios providente, dotado de una presciencia infalible, ¿cómo salvar el azar y no dejarlo morir en la serie causal de los fenómenos? Es el mismo problema de la libertad: si Dios tiene presciencia infalible de todas las cosas, ¿cómo puede haber libertad en la serie fatal de lo que tiene que pasar de acuerdo a la infalibilidad de Dios?

"Si Dios prevé todas las cosas y no puede equivocarse, habrá de suceder cuanto la Providencia ha previsto que suceda. Por tanto si desde toda la eternidad prevé no solo los actos sino también los pensamientos y los deseos, no existe el libre arbitrio". (V, III, 6).

Al primer problema visto arriba se le suma un segundo: si ello que es contingente puede no ser, ¿cómo puede ser previsto por Dios sin que se haga necesario? Y si lo contingente es incierto, ¿cómo puede Dios prever lo incierto sin hacerlo cierto?

"El problema es por tanto: ¿cómo puede Dios prever las cosas que han de suceder, si son inciertas?" (V, III, 65).

Si no hay libertad como quieren estas objeciones demostrar, se siguen graves consecuencias, no solo que la doctrina de la libertad propuesta es pura fantasía, sino también porque entonces los hombres "son llevados al bien o al mal no por la propia voluntad, sino por una incoercible necesidad de lo que ha de suceder". También, en esta hipótesis no tiene sentido la oración y la esperanza, reconocidas aquí por Boecio como medios comunicantes con Dios.

Según Boecio la objeción de que lo contingente e incierto es imposible porque se anula con la presciencia divina que hace todo necesario (objeción ante la cual Cicerón había sucumbido), nace del malentendido de asociar la necesidad de la causalidad de los fenómenos con la presciencia de Dios. Boecio cree poder demostrar que no se asocian necesariamente, y que al contrario, la presciencia divina puede conocer lo incierto como incierto y propone la demostración siguiente: si los signos indican lo que representan, y la Providencia puede tomarse como signo de ello, entonces ella será signo de que todo es necesario o de que no todo es necesario.

(21) Cf. n.4

(22) "Podemos, pues, definir el azar como un hecho o acontecimiento inesperado, producto de la conjunción de causas que actúan en la realización de un fin. La conjunción y coincidencia de causas procede del orden inmutable del universo, que tiene su origen en la Providencia y ordena todas las cosas en su tiempo y lugar". (V, I, 55). Cf., sin embargo, Aristóteles, Física II, 4-6. 
La realidad, lo sabemos, informa que no todo sucede por necesidad, ya que hay hechos que se prevén hacer, pero su realización es libre, incierta, ya que se podrían no hacer. Por ejemplo, cuando uno se prepara para combatir, se prepara porque el resultado no es necesario. Luego, no todo es necesario y la Providencia será signo de ello. Luego, libertad y presciencia divina pueden ser compatibles (23).

Ahora bien, tal como las objeciones muestran, una cosa es que la presciencia y la libertad no sean compatibles y otro es cómo puede la presciencia conocer lo incierto como incierto si ella es infalible. Boecio también aborda este problema como un malentendido. Pero ahora lo soluciona con un principio que se le atribuye a Yámblico. Dice así el principio: "todo lo que se conoce se entiende no según su naturaleza, sino según la capacidad del sujeto cognoscente" (24). Esto quiere decir que la presciencia divina conoce lo futuro contingente, lo incierto, lo libre, según su naturaleza y realidad propia, no la del objeto. Y Dios es eterno, dice Boecio, en el sentido no de perpetuidad (que abarca recorriendo todos los instantes de tiempo), sino como "posesión total y perfecta de una vida interminable" (25), tal que puede abarcar y comprender en forma presente toda la plenitud del tiempo en un presente eterno, porque no es anterior a las cosas creadas por razón del tiempo, sino más bien en razón de la simplicidad de su misma naturaleza.

Por tanto, Dios conoce lo futuro contingente, lo incierto y la acción libre del hombre, "en forma presente tal como sucederán un día como hechos futuros" (26), sin alterar su condición de contingente e incierto, aunque "el mismo hecho futuro, considerado en relación con la presciencia divina, aparecerá como necesario y completamente libre e independiente en su misma naturaleza” (27). -Aquí surge la idea de necessitate ex hypothesi, muy importante en el tratamiento del problema por Santo Tomás de Aquino y Leibniz-.

Por tanto, la inteligencia de Dios ve en un eterno presente, en la simplicidad de su ser, la serie de eventos temporales, ya sean los necesarios o los contingentes. Ello significa que los cambios de propósito de los hombres no sorprenden ni hacen falible a la presciencia de Dios, la cual, como se dijo, no ve lo que ocurre en la serie del tiempo, sino todo lo singular, lo presente, pasado y futuro, como presente eterno, de tal suerte que ya sabe que cambiaremos de opinión y lo que definitivamente haremos: pero no porque ello esté predeterminado a suceder por necesidad, sino porque "todo acontecimiento futuro va precedido por la mirada de Dios que lo atrae y reclama a su siempre actual conocimiento" (28).

\section{SOLUCIÓN FINAL: RAZÓN Y ORACIÓN}

En la Consolatio no vemos a Boecio uniendo las dos partes de su doctrina sobre la libertad humana, es decir, la doctrina positiva que define al hombre como

(23) Consolatio V, IV, 50

(24) Consolatio V, IV, 75

(25) Consolatio V, VI, 10

(26) Consolatio V, VI, 80

(27) Consolatio V, VI, 100.

(28) Consolatio V, VI, 151. 
libre por ser racional y capaz de contemplar la mente divina con las soluciones que hacen compatible la presciencia con la contingencia y la libertad y cómo la presciencia no hace cierto lo incierto que conoce. Puede suponerse, claro está, que si las dos objeciones presentadas han sido refutadas, la doctrina inicial sigue siendo válida.

Así, lo que nos dice Boecio es que no hay razón para negar la libertad humana en el sentido de que ella se anule por un determinismo metafísico, pero no se es verdaderamente libre sin acercarnos a Dios por medio de aquello que es lo mejor en nosotros y común con el bonus rector, a saber, la razón. Ello pone como límite de la libertad humana no a la formalidad de una ley o principio ajeno a la razón, sino a esta misma y al bien que la mente infinita posee por causa de su razón infinita. Es por esto que puede decirse que mientras para el hombre el bien infinito de Dios está sobre su libertad, en Dios coinciden.

"Un dios provisor contempla desde arriba todas las cosas. Y la siempre presente eternidad de su mirada coincide con la futura calidad de nuestros actos, premiando a los buenos y castigando a los malos". (V, VI, 166).

Conforme entonces a lo que nos dice Boecio, la razón es el medio que nos lleva a la libertad porque nos lleva a Dios como fin de la razón, o sea, como verdad. Ello significa, como dijimos, que ningún objeto, máxima subjetiva, o ley formal (que no tiene por qué identificarse con la razón, pudiendo ser esta una interpretación parcial de la razón), hace al hombre realmente libre, sino el dios verdadero que entendemos, que nos es entregado por la razón.

Es, pues, por esta misma noción de razón, que se entiende por qué Boecio puede compartir con los estoicos el que no se es libre si se deja uno a las pasiones que embotan la razón, que es el principio de la libertad, pero ir más allá que ellos, sin embargo, al afirmar que la razón divina no solo se halla en la naturaleza, expresada en la causalidad y el fatalismo de las consecuencias una vez que se han efectuado las causas, sino que expresada en la persona divina que es eternamente atenta al devenir de los sucesos, los necesarios como los contingentes. Así, como consecuencia, desde la noción de razón de Boecio en la Consolatio se sigue la noción de Dios persona, y el sentido de la oración como una actividad que se impone sobre el estado de ánimo ataráxico de los estoicos. En la oración, cree Boecio, compartimos la eternidad del presente con Dios y tenemos injerencia sobre el futuro.

"No es vana, entonces, nuestra esperanza en Dios, ni nuestras oraciones inútiles, pues, si son rectas, no pueden ser ineficaces. Dejad, pues, los vicios: practicad las virtudes. Levantad vuestros corazones a la más alta esperanza y dirigid al cielo vuestras humildes oraciones. Tenéis sobre vosotros una gran necesidad, si no queréis engañaros a vosotros mismos: la necesidad de ser buenos, pues vivís bajo la mirada del juez que todo lo ve”. (V, VI, 170) (29).

(29) Este artículo fue posible gracias al VI Seminario de Estudios Patrísticos, Pontificia Universidad Católica de Chile, Facultades de Teología y Filosofía, septiembre de 2001. El autor de este artículo agradece a los organizadores su invitación, y los comentarios y sugerencias hechas durante la exposición de este trabajo por distinguidos profesores, especialmente a R.P. Sergio Zañartu, de la Facultad de Teología de la Pontificia Universidad Católica de Chile. 


\title{
RESUMEN
}

En este artículo, me propongo mostrar que la discusión de Boecio sobre la compatibilidad de la libertad humana y la praescientia divina (De consolatione philosophiae, Libro V) es una elaborada doctrina que une elementos neoplatónicos y lógica aristotélica. Según argumento, la discusión está conectada a la preocupación anterior sobre este tema mantenida por los estoicos, y sus principales líneas de desarrollo van a influir en pensadores como Santo Tomás de Aquino, L. Valla y G.W. Leibniz. En lo esencial, la doctrina de Boecio dice que no es la sola razón humana lo que hace al hombre libre, sino su fin, que es Dios como ser eternamente presente a los procesos necesarios y contingentes del universo. Esto está supuesto en sus palabras finales sobre el poder de la oración.

\begin{abstract}
In this article, I attempt to show that Boethius' discussion on the compatibility between human freedom and praescientia divina (De consolatione philosophiae, Book V) is a refined piece of doctrine combining Neoplatonic elements and Aristotelian logic. The discussion, I argue, is connected to the Stoic early concern on the topic and its main lines of development are to be influential in later thinkers as St. Th. Aquinas, L. Valla and G.W. Leibniz. Basically, Boethius' doctrine means that it is not only the human reason by itself what makes the man free, but also the end of reason, i.e., God, who is eternally present to necessary and contingent processes in the universe. This is suggested by his final words on the power of prayer.
\end{abstract}

